\title{
ESTUDIO EXPERIMENTAL DE LA ADICIÓN DE CENIZA DE BAGAZO DE CAÑA COMO FILLER PARA PRODUCIR CONCRETOS AUTOCONSOLIDANTES
}

\author{
J. A. ZEA, F. A. ALONSO, A. LÓPEZ, J. E. MANDUJANO \\ Facultad de Ingeniería, Universidad Autónoma de Chiapas, México
}

\begin{abstract}
RESUMEN
La demanda y consumo de cemento han llevado a la búsqueda de materiales alternativos y no convencionales para su uso eficiente, y al mismo tiempo que contribuyan al aprovechamiento, manejo y mitigación de desechos. Este estudio presenta la influencia del uso de filler de ceniza de bagazo de caña (CBC), como residuo agroindustrial adicionándose en proporciones de $0 \%, 5 \%, 10 \%, 15 \%, 20 \%$ y $25 \%$ respecto al peso de cemento para la elaboración de concretos autoconsolidantes (CAC). El proceso experimental ha sido satisfactorio, teniendo resultados y desempeños excelentes en proporciones de $10 \%, 15 \%$ y $20 \%$, los cuales tendrán que compararse entre sí de acuerdo a los resultados obtenidos en cuanto a sus parámetros de trabajabilidad y resistencia a compresión.
\end{abstract}

Palabras clave: Autoconsolidante, Ceniza, Agroindustrial, Adición, Filler.

\begin{abstract}
The demand and uptake of cement have led to the search for alternative and non-conventional materials for efficient use, and at the same time that contribute to the use, management and mitigation of waste. This study present the influence of the use cane bagasse ash filler (CBA), as the agroindustrial term adding in proportions of $0 \%, 5 \%, 10 \%, 15 \%, 20 \%$ and $25 \%$ with respect to the weight of cement for the production of self-compacting concrete (SCC). The experimental process has been satisfactory, having excellent results and performances in proportions of $10 \%, 15 \%$ and $20 \%$, which will have to be compared with each other according to the results obtained in terms of their workability and compression resistance parameters.
\end{abstract}

Keywords: Self-Consolidation; Ash; Agroindustrial; Addition; Filler. 


\section{INTRODUCCIÓN}

La gestión ambiental y el impulso al desarrollo sostenible son cada vez más rutinarios en los últimos años, la conciencia colectiva ha ido en aumento con respecto a los problemas potenciales que genera el crecimiento de la industria de la construcción en el deterioro del medio ambiente. En términos generales, la construcción no es una actividad amigable con el medio ambiente, ya que genera efectos sobre la tierra, agotamiento de recursos naturales, generación de residuos y diversas formas de contaminación como son las emisiones antropogénicas de $\mathrm{CO}_{2}$ (Hill, Bowen 1997, Buildings Department, 2006, Habert et al, 2012, Wadel, 2014).

No se puede negar el papel que ha jugado el cemento en la historia de la humanidad y sobre todo el cemento portland, creado a principios del Siglo XIX, sin embargo, en las últimas décadas se han suscitado algunos cuestionamientos al empleo de este material, asociados a su alto costo y al impacto ambiental que genera su producción y uso extensivo. También es notable el incremento de la producción de cemento que han experimentado los países del tercer mundo en las últimas décadas.

La producción del cemento es en gran cantidad en la República Mexicana compuesta por varias empresas dedicadas a su producción. La problemática en la que nos encontramos es que en la producción del cemento se generan grandes cantidades de dióxido de carbono $\left(\mathrm{CO}_{2}\right)$, ya que por cada tonelada de Cemento Portland Compuesto (CPC) producida se emite el 30\% de esta a la atmósfera. Tan solo en el año 2006 se produjeron 37.9 millones de toneladas de CPC; de las cuales se emitieron 11.37 millones de toneladas de $\mathrm{CO}_{2}$ a la atmósfera, que es uno de los principales contribuyentes para el calentamiento global.

El alto consumo de recursos naturales, energéticos y las emisiones de $\mathrm{CO}_{2}$, y otros contaminantes (Cachán, 2001) comprometen las posibilidades futuras de utilización de este maravilloso material de construcción. Para atenuar esta situación se trabaja en la disminución de su impacto ambiental, por dos vías; 1) la de mejorar la eficiencia de los procesos de producción en planta, con el objeto de disminuir el consumo energético y 2) optimizar el consumo del cemento.

Este trabajo se enmarca en la segunda vía apoyado en numerosas experiencias y avances que ha experimentado la tecnología del concreto en las últimas décadas, potenciados por la utilización de modernos equipos de visualización y evaluación de materiales de gran finura como el cemento (Martirena, et al 1997) y la aparición de potentes aditivos químicos, adiciones minerales, adiciones naturales y materiales alternativos que han ampliado considerablemente las posibilidades para disminuir la proporción de cemento en la mezcla sin afectar e incluso mejorando las propiedades del concreto (Mehta, 2000, Nasvik, 2006).

En todo el mundo se están llevando a cabo planes para controlar y regular la supervisión de subproductos, residuos y desechos industriales con el fin de preservar el medio ambiente. Existe una gran generación de desechos agroindustriales a los cuales como solución inmediata son incinerados, lo que resulta en una buena solución en su reducción de volumen y con ello se resuelve en parte el problema del reciclaje. La caña de azúcar es uno de los principales cultivos en varios países y toda su producción es más de 1500 millones de toneladas (Dharanidharan, et al 2015). Particularmente en México se cuenta con una producción nacional de aproximadamente 56 millones de toneladas (SIAP, 2018).

Actualmente la industria azucarera está conformada por 54 ingenios, distribuidos en 15 estados y 267 municipios de nuestro País. Particularmente el Estado de Chiapas ocupa el $5^{\circ}$ lugar nacional en producción que representa el 5.5\% del total aportado (SIAP, 2018), y se cuenta con dos ingenios: "Belisario Domínguez y Pujiltic" ubicados en los municipios de Huixtla y Venustiano Carranza respectivamente, donde la generación de residuos en las unidades productivas constituye una de las principales fuentes de contaminación de esta industria, junto con las descargas de aguas residuales y las emisiones a la atmósfera. 
La utilización de algunas cenizas de desechos agrícolas proporciona ventajas al concreto tanto en estado fresco como endurecido, así como la mejora de su durabilidad. Entre las ventajas adicionales de su uso se encuentra la reducción de costos de producción (optimización del cemento) y reducción de los problemas ambientales que se asocian con su manejo y disposición. Es por ello que en este trabajo se analizó la influencia de la adición como filler de la ceniza de bagazo de caña (CBC) en estado fresco y endurecido para la elaboración de concretos autoconsolidantes (CAC).

\section{PROCEDIMIENTO}

El objetivo central de esta investigación es la de evaluar la adición de la Ceniza de Bagazo de Caña (CBC) como filler en el comportamiento del Concreto Autoconsolidante (CAC) en estado fresco y endurecido, para ello se implementarán una serie de procesos que inician desde la caracterización de los materiales componentes así como de la adición de la ceniza de bagazo de caña, la trabajabilidad del concreto hidráulico de control y las mezclas con diferentes proporciones de CBC, la aplicación de diferentes métodos de ensayo para concretos autoconsolidantes y el análisis de las propiedades mecánicas de los mismos.

\subsection{Proporcionamientos requeridos}

\subsubsection{Diseño experimental}

Para este estudio se realizaron siete mezclas en las que se únicamente se varió el contenido de ceniza de bagazo de caña (CBC) en cada una $(5,10,15,20$ y 25\%) (Tabla 1), esto para un agregado grueso de tamaño máximo de $12.7 \mathrm{~mm}(1 / 2$ ”), así mismo se elaboró la mezcla de control y una mezcla adicional de tipo fluida en la que únicamente se le adicionó el aditivo superfluidificante y conocer su comportamiento. Todas las mezclas se diseñaron de acuerdo con el método de volúmenes absolutos del Instituto Americano del Concreto (ACI-211.1, 2002), para lo cual se consideró una relación agua/cemento de 0.50 .

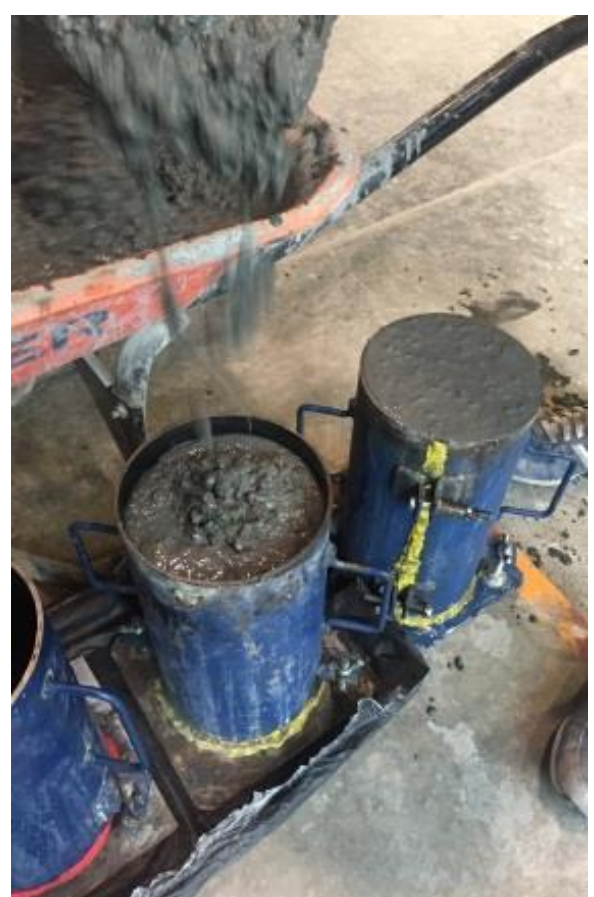

Figura 1.- Llenado de cilindros con concreto autoconsolidante Fuente: Toma propia. 
Tabla 1.- Diseño de Mezclas de Concreto

\begin{tabular}{|c|c|c|c|c|c|c|c|}
\hline $\begin{array}{l}\text { Diseño de } \\
\text { mezcla }\end{array}$ & $\begin{array}{c}\text { Relación } \\
\text { a/c }\end{array}$ & $\begin{array}{c}\text { Agua } \\
\left(\mathrm{kg} / \mathrm{m}^{3}\right)\end{array}$ & $\begin{array}{c}\text { Cemento } \\
\left(\mathrm{kg} / \mathrm{m}^{3}\right)\end{array}$ & $\begin{array}{c}\text { Ceniza de } \\
\text { bagazo de } \\
\text { caña } \\
\left(\mathrm{kg} / \mathrm{m}^{3}\right)\end{array}$ & $\begin{array}{l}\text { Agregado } \\
\text { fino } \\
\left(\mathrm{kg} / \mathrm{m}^{3}\right)\end{array}$ & $\begin{array}{l}\text { Agregado } \\
\text { grueso } \\
\left(\mathrm{kg} / \mathrm{m}^{3}\right)\end{array}$ & $\begin{array}{c}\text { Superfluidificante } \\
\text { (\% por peso } \\
\text { de cemento) }\end{array}$ \\
\hline CC-0\% CBC & 0.50 & 190.00 & 380.00 & --- & 830.00 & 845.00 & 2.00 \\
\hline CF-0\% CBC & 0.50 & 190.00 & 380.00 & --- & 830.00 & 845.00 & 2.00 \\
\hline $\mathrm{CAC}-15 \% \mathrm{CBC}$ & 0.50 & 190.00 & 380.00 & 57.00 & 830.00 & 845.00 & 2.00 \\
\hline $\mathrm{CAC}-20 \% \mathrm{CBC}$ & 0.50 & 190.00 & 380.00 & 76.00 & 830.00 & 845.00 & 2.00 \\
\hline CAC-25\% CBC & 0.50 & 190.00 & 380.00 & 95.00 & 830.00 & 845.00 & 2.00 \\
\hline
\end{tabular}

Fuente: Elaboración propia

\subsubsection{Preparación de probetas}

Para este estudio se prepararon en total 110 cilindros para las pruebas de resistencia a compresión. Después de fabricar las probetas se cubrieron por $24 \mathrm{~h}$; posteriormente se desmoldaron y recibieron un curado por inmersión en una solución saturada de hidróxido de calcio hasta la fecha de ensaye. Las edades de ensayo y el número de probetas para cada prueba considerada se muestran en la Tabla 2.

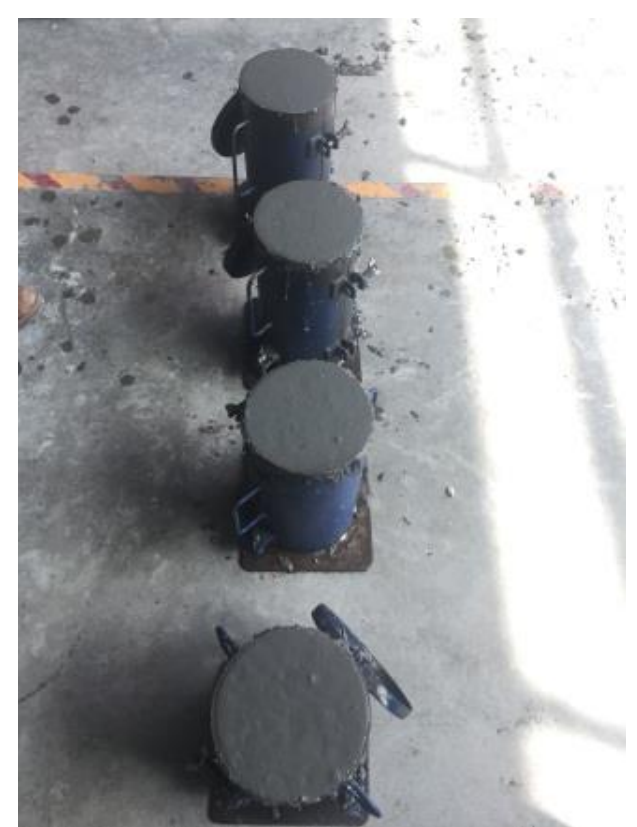

Figura 2.- Preparación de cilindros para pruebas

Fuente: Toma propia.

Tabla 2.- Características de las probetas y tiempos de prueba para ensayos mecánicos

\begin{tabular}{|cccc|}
\hline Prueba & Descripción & $\begin{array}{c}\text { Edad de prueba } \\
(\text { días })\end{array}$ & $\begin{array}{c}\text { Número de } \\
\text { Cilindros }\end{array}$ \\
\hline $\begin{array}{c}\text { Resistencia a } \\
\text { compresión }\end{array}$ & $\begin{array}{c}\text { Cilindros: } 150 \mathrm{~mm} \varnothing \times \\
\text { x }\end{array}$ & $\begin{array}{c}7,14,28,56,90, \\
180\end{array}$ & 110 \\
\hline
\end{tabular}

Fuente: Elaboración propia 


\subsubsection{Caracterización de las mezclas en estado fresco}

Las diversas mezclas se prepararon en el Laboratorio de la Facultad de Ingeniería de la UNACH conforme a las "Especificaciones y directrices para el Hormigón autoconsolidante - HAC" de EFNARC (European Federation of National Associations Representing for Concrete) (EFNARC, 2002).
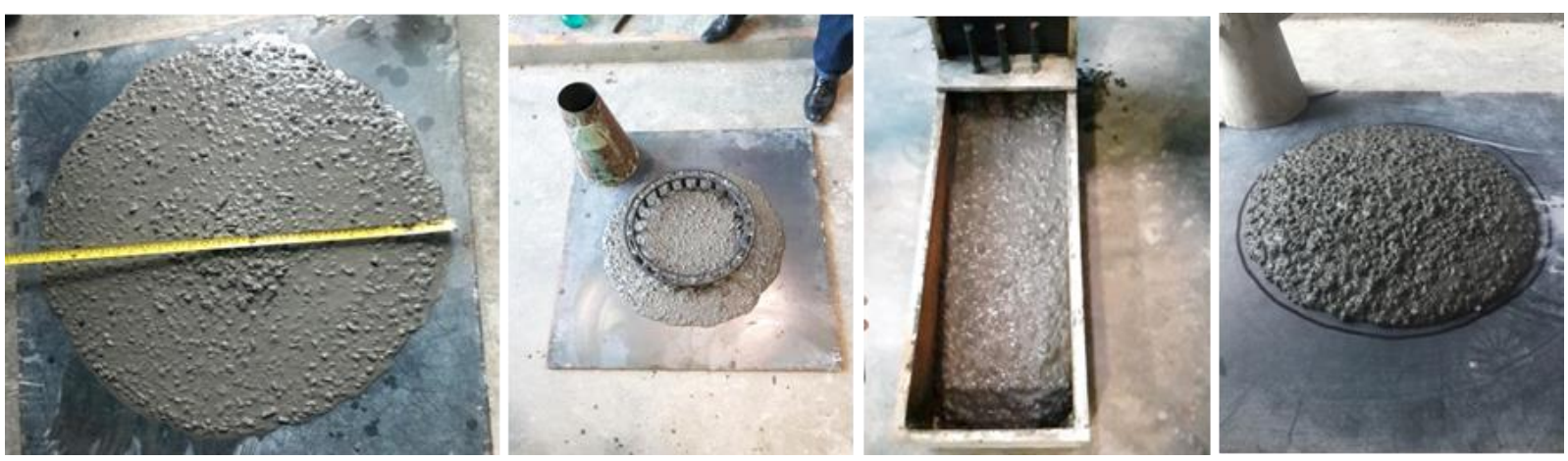

Figura 3.- Caracterización de mezclas en estado fresco para la evaluación de los parámetros de trabajabilidad elementales de un CAC.

Fuente: Toma propia.

\subsubsection{Caracterización de las mezclas en estado endurecido}

\subsubsection{Resistencia a la compresión}

Las pruebas de resistencia a la compresión se realizaron de acuerdo con la norma ASTM C 39-4 (Standard Test Method for Compressive Strength of Cylindrical Concrete Specimens) (ASTM, 2004). En total se emplearon 110 especímenes.
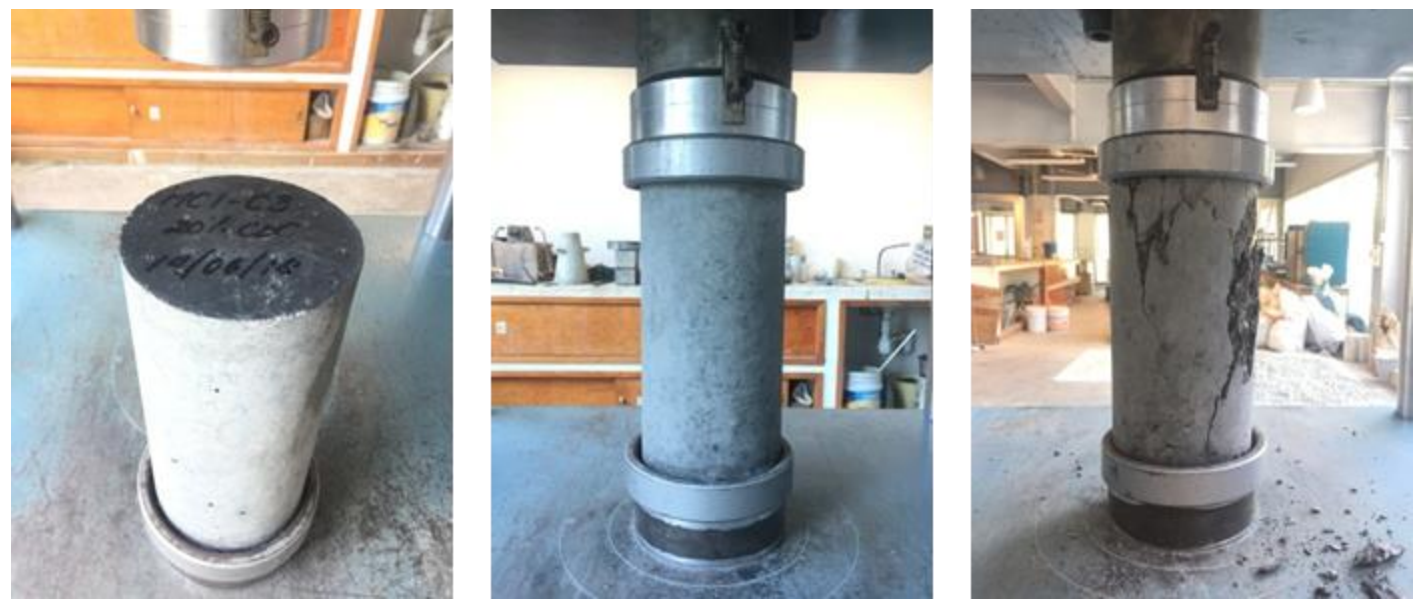

Figura 4.- Prueba de resistencia a compresión (Izquierda a derecha: Identificación de probetas, colocación de probeta, probeta ensayada)

Fuente: Toma propia.

\section{RESULTADOS}

\subsection{Composición química de las adiciones minerales}

En general, la espectroscopia IR identifica aceptablemente la naturaleza de los grupos $\mathrm{OH}$, y las distintas formas en las que el agua puede estar integrada en un compuesto. Una ventaja adicional no despreciable es su carácter prácticamente no destructivo, dado que para realizar las determinaciones se precisan cantidades muy pequeñas de muestra. Como se describió en líneas anteriores, el FTIR ofrece análisis tanto cualitativos como cuantitativos de una amplia gama de muestras orgánicas e inorgánicas, así mismo podemos obtener datos espectrales de alta calidad, pruebas de control de calidad / control de 
calidad rutinarias o necesidades de investigación. La figura siguiente se muestra el espectro FTIR de la ceniza de bagazo de caña (CBC) y cemento portland. El análisis se realizó en el infrarrojo medio en la zona vibracional de 4000 a $600 \mathrm{~cm}^{-1}$, ya que presentan similitudes en algunos picos y se busca dar énfasis en el uso de las cenizas como material adicionante.

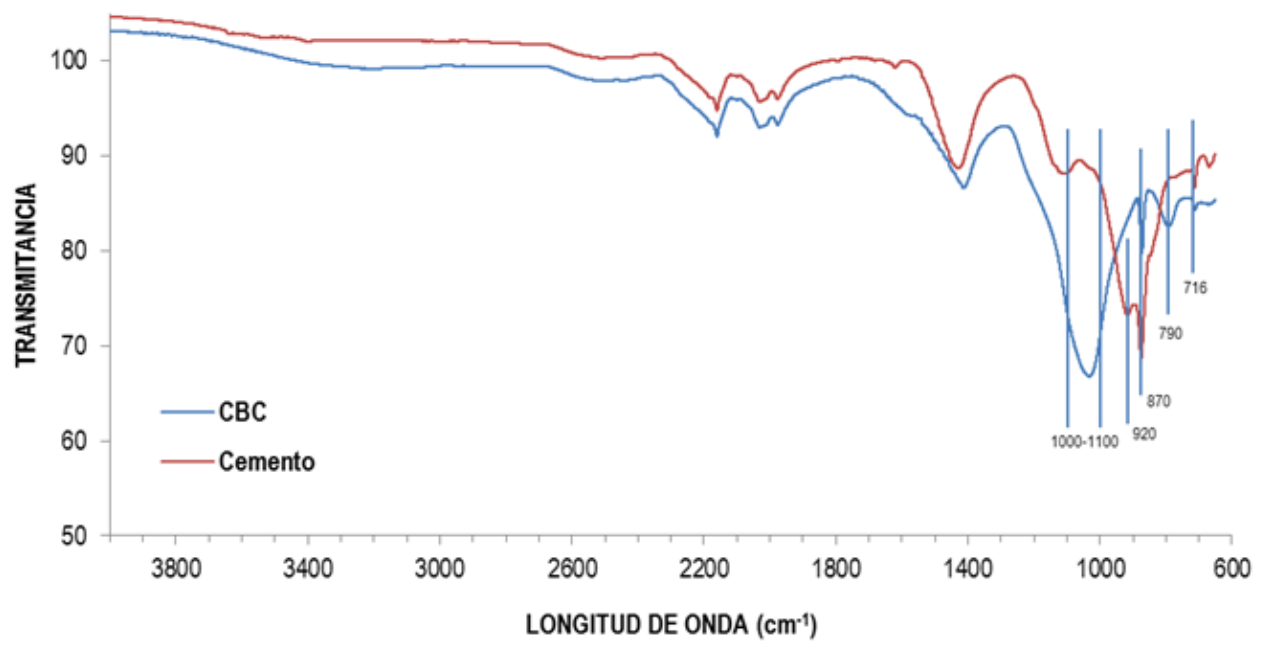

Figura 5.- Espectro FTIR comparativo de la CBC y el CPC 30R

Fuente: Elaboración propia.

En la interpretación de los espectros, es importante señalar que la estructura de los silicatos y aluminosilicatos, componentes principales de las cenizas volantes y cemento, en general está constituida por unidades cíclicas que se forman por la conexión de tetraedros de aluminio y silicio a través de puentes de oxígeno, con diferente número de miembros (Kovalchuk, Fernández-Jiménez, 2008, Criado, et al 2007).

\subsection{Caracterización física y química del cemento y la ceniza de bagazo de caña}

En la tabla siguiente se presentan las características químicas de la ceniza y del cemento usado para los respectivos ensayos de adición al mismo. De acuerdo a la tabla, los compuestos con mayor presencia en la CBC son $\mathrm{SiO}_{2}, \mathrm{CaO}$ y $\mathrm{Fe}_{2} \mathrm{O}_{3}$. Según la norma ASTM C618 (Standard Specification for Coal Fly Ash and Raw or Calcined Natural Pozzolan for Use in Concrete) ( ASTM, 2019) en las puzolanas clase $\mathrm{N}$, se presentan composiciones de $\mathrm{SiO}_{2}, \mathrm{Al}_{2} \mathrm{O}_{3}$ y $\mathrm{Fe}_{2} \mathrm{O}_{3}$, donde la suma de éstos debe ser igual o superior al 70\%. Diversas investigaciones han reportado que la suma de estos tres compuestos representa más del 70\% de la composición química de la ceniza de bagazo de caña (Rincón, et al 2010), sin embargo en las cenizas obtenidas para este proyecto presentaron muy bajo contenido de alúmina $\left(\mathrm{Al}_{2} \mathrm{O}_{3}\right)$.

Tabla 3.- Características químicas de la CBC y el CPC 30R

\begin{tabular}{|ccc|}
\hline Características (\%) & CBC & Cemento \\
\hline $\mathbf{S i O}_{\mathbf{2}}$ & 55.1 & 24.3 \\
\hline $\mathbf{A l}_{\mathbf{2}} \mathbf{O}_{\mathbf{3}}$ & 0.7 & 4.3 \\
\hline $\mathbf{F e}_{\mathbf{2}} \mathbf{O}_{3}$ & 1.0 & 3.0 \\
\hline $\mathbf{C a O}$ & 39.7 & 58.8 \\
\hline $\mathbf{M g O}$ & 1.1 & 1.4 \\
\hline $\mathbf{K}_{\mathbf{2}} \mathbf{O}$ & 0.8 & 0.7 \\
\hline $\mathbf{N a}_{2} \mathbf{O}$ & 0.9 & 0.8 \\
\hline
\end{tabular}

Fuente: Elaboración propia 
La pérdida al fuego para las muestras obtenidas en este trabajo, son altas, lo cual se atribuye a que corresponde a una muestra tomada de un vertedero a cielo abierto y la alta posibilidad donde se utiliza carbón para el proceso de quema. Algunos autores indican que la pérdida por ignición puede disminuirse sometiendo las muestras a procesos térmicos donde se incremente la temperatura de quema (Cordeiro, et al 2009).

\subsection{Composición mineralógica}

La composición mineralógica de la ceniza fue evaluada por la técnica de Difracción de Rayos X (DRX), con un equipo marca BRUKER a una velocidad de escaneo de $5 \% \mathrm{~min}$ con un paso de 0.020 grados. En la siguiente se presenta el difractograma. Se observa que la ceniza presenta características amorfas debido al levantamiento de la línea base entre 15 y 35 (20); esta amorficidad le confiere actividad puzolánica a la ceniza, que la hace apta para ser adicionada al cemento portland (Martirena, et al 1998). Se destaca la presencia de cristobalita y cuarzo (ambas estructuras amorfas de $\mathrm{SiO}_{2}$ ), este último se identifica como la fase cristalina predominante; los resultados obtenidos coinciden con los presentados por diferentes autores (Ganesan, et al 2007).

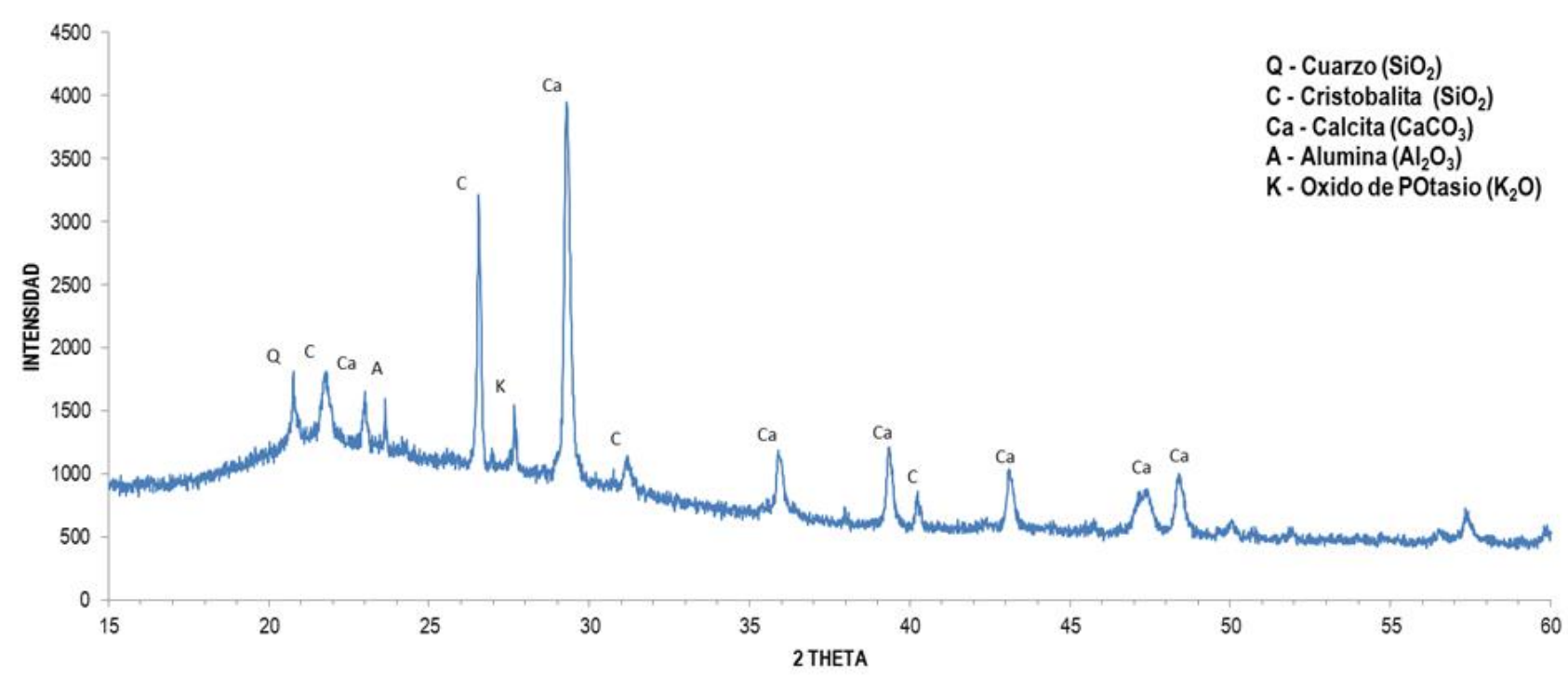

Figura 6.- Difractograma de la CBC obtenida en nuestra región.

Fuente: Elaboración propia.

\subsection{Propiedades en estado fresco de los concretos autoconsolidantes}

Los resultados obtenidos de las pruebas realizadas en estado fresco para las diferentes mezclas establecidas de concretos autoconsolidantes se reportan en las Tablas siguientes. Las pruebas en estado fresco se llevaron a cabo conforme a las "Especificaciones y directrices para el Hormigón autoconsolidante - HAC" de EFNARC (European Federation of National Associations Representing for Concrete) (EFNARC, 2002) para el ensayo de Flujo de asentamiento y ensayo de $\mathrm{T}_{50 \mathrm{~cm}}$, ensayo de Anillo en J, método de ensayo de caja en L, ensayo de embudo V y ensayo de embudo $\mathrm{V}$ a $\mathrm{T}_{5 \text { minutos }} \mathrm{y}$ para la obtención del Índice de Estabilidad Visual se tomó en cuenta la norma ASTM C1611 (Standard Test Method for Slump Flow of Self-Consolidating Concrete) (ASTM, 2018). 
Tabla 4.- Resumen de Ensayo de flujo de asentamiento y ensayo de $\mathrm{T}_{50 \mathrm{~cm}}$

\begin{tabular}{|ccccccc|}
\hline Mezcla & $\begin{array}{c}\mathrm{D}_{1} \\
(\mathrm{~cm})\end{array}$ & $\begin{array}{c}\mathrm{D}_{2} \\
(\mathrm{~cm})\end{array}$ & $\begin{array}{c}\mathrm{D}_{\mathrm{f}} \\
(\mathrm{cm})\end{array}$ & $\begin{array}{c}\text { Flujo asentamiento } \\
\text { por cono de Abrams } \\
\text { (EFNARC, S., 2002) }\end{array}$ & $\begin{array}{c}\mathrm{T}_{50} \\
(\mathrm{~s})\end{array}$ & $\begin{array}{c}\text { Flujo asentamiento } \\
\mathrm{T}_{50 \mathrm{~cm}}\end{array}$ \\
(EFNARC, $\mathrm{S} ., 2002)$
\end{tabular}

Fuente: Elaboración propia.

Tabla 5.- Resumen de Ensayo de anillo J

\begin{tabular}{|c|c|c|c|c|c|c|c|c|c|}
\hline Mezcla & $\begin{array}{l}\mathrm{D}_{1} \\
(\mathrm{~cm})\end{array}$ & $\begin{array}{c}\mathrm{D}_{2} \\
(\mathrm{~cm})\end{array}$ & $\begin{array}{c}\mathrm{D}_{\mathrm{f}} \\
(\mathrm{cm})\end{array}$ & $\begin{array}{c}\mathrm{H}_{1} \\
\text { (Interior) } \\
(\mathrm{cm})\end{array}$ & $\begin{array}{c}\mathrm{H}_{2} \\
\text { (Exterior) } \\
(\mathrm{cm})\end{array}$ & $\begin{array}{c}\mathrm{A}_{1} \\
(\mathrm{~cm})\end{array}$ & $\begin{array}{c}\mathrm{A}_{2} \\
(\mathrm{~cm})\end{array}$ & $\begin{array}{l}\text { Diferencia } \\
\text { de alturas } \\
(\mathrm{cm})\end{array}$ & $\begin{array}{c}\text { Diferencia } \\
\text { de Alturas } \\
\text { (EFNARC, S., } \\
\text { 2002) }\end{array}$ \\
\hline $\begin{array}{l}\text { Concreto de } \\
\text { Control }\end{array}$ & --- & --- & --- & --- & --- & --- & --- & --- & \multirow{7}{*}{$\begin{array}{l}\text { Mínimo: } 0 \mathrm{~mm} \\
\text { Máximo: } 10 \mathrm{~mm}\end{array}$} \\
\hline Concreto Fluido & 52.0 & 54.0 & 53.0 & 8.0 & 11.0 & 4.0 & 1.0 & 3.0 & \\
\hline $\mathrm{CAC}-5 \% \mathrm{CBC}$ & 48.0 & 51.0 & 49.5 & 8.0 & 11.0 & 4.0 & 1.0 & 3.0 & \\
\hline CAC - $10 \%$ CBC & 64.0 & 65.0 & 64.5 & 8.0 & 10.0 & 4.0 & 2.0 & 2.0 & \\
\hline CAC - $15 \%$ CBC & 47.0 & 47.0 & 47.0 & 8.5 & 11.0 & 3.5 & 1.0 & 2.5 & \\
\hline CAC - $20 \%$ CBC & 59.0 & 57.0 & 58.0 & 10.0 & 11.0 & 2.0 & 1.0 & 1.0 & \\
\hline CAC - $25 \%$ CBC & 69.0 & 68.0 & 68.5 & 9.5 & 11.0 & 2.5 & 1.0 & 1.5 & \\
\hline
\end{tabular}

Fuente: Elaboración propia.

Tabla 6.- Resumen de Ensayo de caja en L

\begin{tabular}{|c|c|c|c|c|c|}
\hline Mezcla & $\begin{array}{l}\mathrm{T}_{60} \\
(\mathrm{~s})\end{array}$ & $\begin{array}{l}\mathrm{H}_{1} \\
(\mathrm{~cm})\end{array}$ & $\begin{array}{c}\mathrm{H}_{2} \\
(\mathrm{~cm})\end{array}$ & $\mathrm{H}_{2} / \mathrm{H}_{1}$ & $\begin{array}{c}\text { Relación } \mathrm{H}_{2} / \mathrm{H}_{1} \\
\text { (EFNARC, S., } \\
\text { 2002) }\end{array}$ \\
\hline $\begin{array}{c}\text { Concreto de } \\
\text { Control }\end{array}$ & --- & --- & --- & --- & \multirow{7}{*}{$\begin{array}{l}\text { Mínimo: } 0.8 \\
\text { Máximo: } 1.0\end{array}$} \\
\hline Concreto Fluido & 42.2 & 23.5 & 11.5 & 0.49 & \\
\hline $\mathrm{CAC}-5 \% \mathrm{CBC}$ & 103.1 & --- & --- & --- & \\
\hline $\mathrm{CAC}-10 \% \mathrm{CBC}$ & 21.2 & 10.0 & 8.5 & 0.85 & \\
\hline $\mathrm{CAC}-15 \% \mathrm{CBC}$ & 11.4 & 14.5 & 3.0 & 0.21 & \\
\hline $\mathrm{CAC}-20 \% \mathrm{CBC}$ & 8.6 & 9.0 & 8.0 & 0.89 & \\
\hline $\mathrm{CAC}-25 \% \mathrm{CBC}$ & 4.2 & 9.0 & 8.0 & 0.89 & \\
\hline
\end{tabular}

Fuente: Elaboración propia. 
Tabla 7.- Resumen de Ensayo de embudo V y embudo V a $\mathrm{T}_{5 \text { minutos }}$

\begin{tabular}{|c|c|c|c|c|}
\hline Mezcla & $\begin{array}{l}\text { Tiempo } \\
\text { de flujo }\end{array}$ & $\begin{array}{c}\text { Embudo V } \\
\text { (EFNARC, S., 2002) }\end{array}$ & $\begin{array}{l}\text { Tiempo de } \\
\text { flujo } \\
T_{5 \text { minutos }} \\
(\mathrm{s})\end{array}$ & $\begin{array}{l}\text { Embudo V a T } 5 \text { minutos } \\
\text { (EFNARC, S., 2002) }\end{array}$ \\
\hline $\begin{array}{l}\text { Concreto de } \\
\text { Control }\end{array}$ & --- & \multirow{7}{*}{$\begin{array}{l}\text { Mínimo: } 6 \text { segundos } \\
\text { Máximo: } 12 \text { segundos }\end{array}$} & --- & \multirow{7}{*}{$\begin{array}{l}\text { Mínimo: } 0 \text { segundos } \\
\text { Máximo: +3 segundos }\end{array}$} \\
\hline Concreto Fluido & 13.8 & & 17.2 & \\
\hline CAC - 5\% CBC & 12.5 & & 14.3 & \\
\hline $\mathrm{CAC}-10 \% \mathrm{CBC}$ & 9.7 & & 12.7 & \\
\hline $\mathrm{CAC}-15 \% \mathrm{CBC}$ & 11.1 & & 12.3 & \\
\hline $\mathrm{CAC}-20 \% \mathrm{CBC}$ & 8.7 & & 11.5 & \\
\hline $\mathrm{CAC}-25 \% \mathrm{CBC}$ & 6.5 & & 9.2 & \\
\hline
\end{tabular}

Fuente: Elaboración propia.

Tabla 8.- Resumen del Índice de Estabilidad Visual

\begin{tabular}{|c|c|c|}
\hline Mezcla & Valor & $\begin{array}{c}\text { Valores para la evaluación del VSI } \\
\text { (ASTM C1611, 2018) }\end{array}$ \\
\hline $\begin{array}{c}\text { Concreto de } \\
\text { Control }\end{array}$ & --- & \multirow{7}{*}{$\begin{array}{l}\text { - Estable. Sin segregación= } 0 \\
\text { - Ligera exudación, sin segregación= } 1 \\
\text { - Ligero halo de mortero }(<1 \mathrm{~cm}) \text {. Exudación notable= } 2 \\
\text { - Halo de mortero }(>1 \mathrm{~cm}) \text { y segregación considerables }=3\end{array}$} \\
\hline Concreto Fluido & 2 & \\
\hline $\mathrm{CAC}-5 \% \mathrm{CBC}$ & 3 & \\
\hline $\mathrm{CAC}-10 \% \mathrm{CBC}$ & 1 & \\
\hline $\mathrm{CAC}-15 \% \mathrm{CBC}$ & 0 & \\
\hline CAC - $20 \%$ CBC & 0 & \\
\hline $\mathrm{CAC}-25 \% \mathrm{CBC}$ & 1 & \\
\hline
\end{tabular}

Fuente: Elaboración propia.

Como se observa de acuerdo a los resultados expresados en las tablas anteriores, la mezcla de concreto autoconsolidante con $20 \%$ de adición de CBC como filler, es la única que se comporta de acuerdo a las especificaciones y directrices para el hormigón autoconsolidante-HAC de EFNARC (EFNARC, 2002) y la norma ASTM C1611 (Standard Test Method for Slump Flow of Self - Consolidating Concrete) (ASTM, 2018).

\subsection{Propiedades en estado endurecido de los concretos autoconsolidantes}

\subsubsection{Resistencia a compresión}

En las figuras siguientes se muestran los resultados de resistencia a compresión a 7, 14, 28, 56 y 90 días para todas las mezclas. Se observa que la resistencia a compresión depende del tipo y porcentaje de adición mineral. En todas las mezclas la resistencia a compresión se incrementa con la edad porque existe suficiente humedad para propiciar las reacciones de hidratación del cemento y las reacciones puzolánicas de la CBC y la CV, tal como se ha reportado en la literatura (Neville, Brooks, 1998), aunque a diferente razón de cambio. También, es posible apreciar el incremento de resistencia debido al efecto de la reacción puzolánica en las mezclas de concreto que contienen adiciones minerales, puesto que a totas las edades son superadas en relación al concreto de control. 
Tabla 9.- Resumen de resultados promedios a compresión a diferentes edades

\begin{tabular}{|cccccc|}
\hline \multirow{2}{*}{ Mezcla } & \multicolumn{5}{c|}{ Edad de prueba } \\
\hline Concreto de Control & 252.10 & 265.96 & 320.62 & 343.58 & 352.19 \\
Concreto Fluido & 297.26 & 335.74 & 339.57 & 350.21 & 362.13 \\
CAC - 5\% CBC & 191.41 & 229.67 & 241.33 & 295.82 & 301.27 \\
CAC - 10\% CBC & 304.79 & 334.69 & 399.63 & 445.42 & 461.06 \\
CAC - 15\% CBC & 279.83 & 322.12 & 377.04 & 413.03 & 421.09 \\
CAC - 20\% CBC & 289.46 & 351.63 & 405.86 & 433.81 & 457.15 \\
CAC - 25\% CBC & 146.27 & 188.62 & 205.71 & 232.35 & 241.23 \\
\hline
\end{tabular}

Fuente: Elaboración propia.

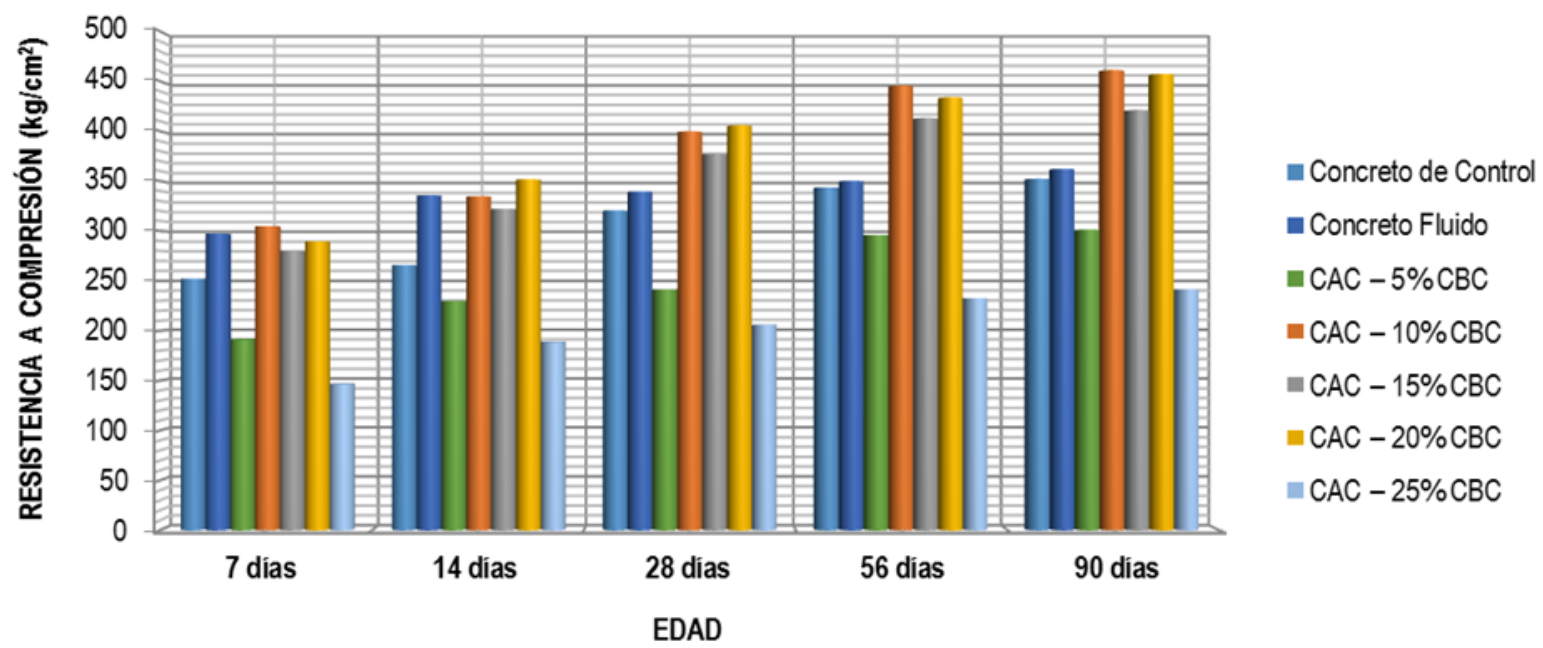

Figura 7.- Valores promedio de resistencia a compresión de las mezclas de concreto autoconsolidante. Fuente: Elaboración propia.

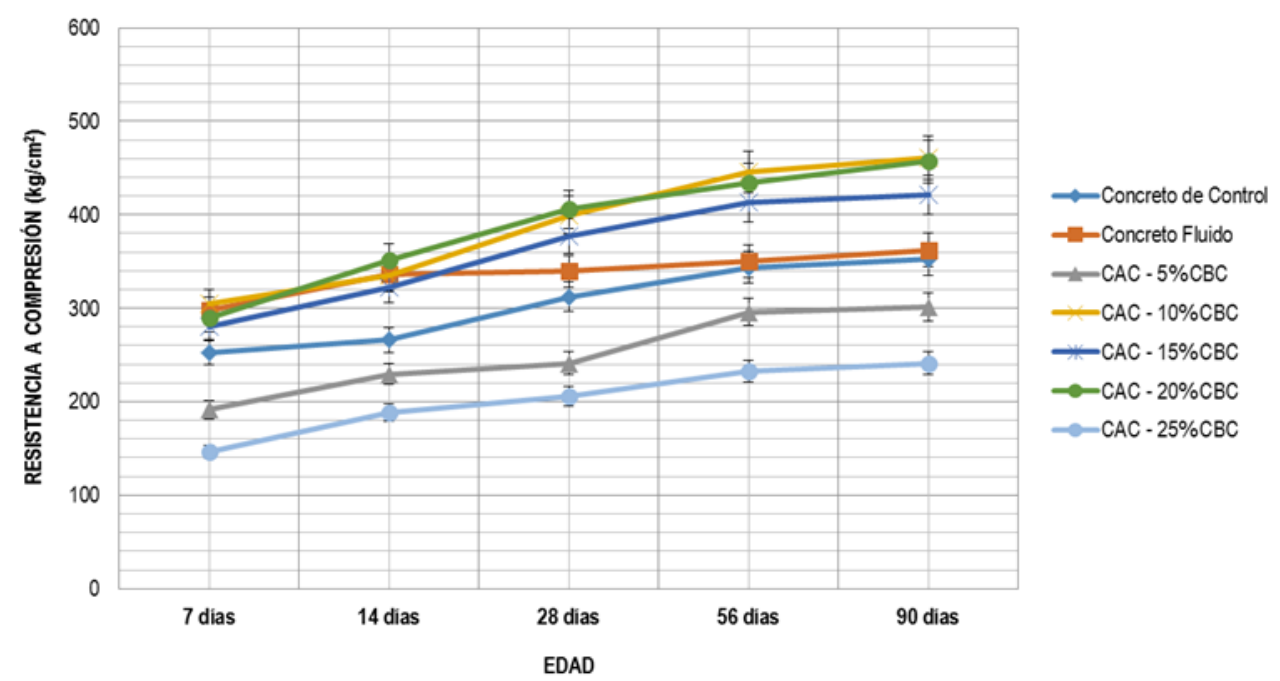

Figura 8.- Estimación de error en valores promedio de resistencia a compresión de las mezclas de concreto autoconsolidante.

Fuente: Elaboración propia. 


\section{RESULTADOS}

- La presente investigación demuestra que es posible diseñar un CAC con materiales de la región incorporando un desecho agroindustrial como la CBC, lo que reduce efectos adversos al medio ambiente.

- En relación con la evaluación de los parámetros de trabajabilidad elementales de un CAC como son: capacidad de relleno, capacidad de paso y resistencia a la segregación, la mezcla con mejor desempeño fue la proporción del $20 \%$ de CBC.

- Respecto a la resistencia a compresión se observa que el uso de CBC como filler representa ventajas favorables, en específico para proporciones del 10, 15 y 20\% de CBC, en donde se incrementó en resistencia última (90 días) hasta en un 30.91, 19.56 y 29.80\% respectivamente en relación a la muestra de control.

- El uso de CBC como filler, permite optimizar el consumo de cemento y modificar la dosificación de la mezcla para emplear relaciones A/C menores a las requeridas.

\section{AGRADECIMIENTOS}

A la Facultad de Ingeniería de la Universidad Autónoma de Chiapas por las facilidades otorgadas al proyecto para su realización y el uso de los diversos laboratorios existentes para llevar a cabo los estudios y ensayos correspondientes.

\section{REFERENCIAS}

American Concrete Institute. (2002). ACI-211.1: Standard Practice for Selecting Proportions for Normal Heavyweight, and Mass Concrete. Michigan, United States of America.

American Society for Testing and Materials. (2004). ASTM-C39/C39M-04a: Standard Test Method for Compressive Strength of Cylindrical Concrete Specimens. West Conshohocken, PA, United States of America: ASTM International.

American Society for Testing and Materials. (2019). ASTM-C618-19: Standard Specification for Coal Fly Ash and Raw or Calcined Natural Pozzolan for Use in Concrete. West Conshohocken, PA, United States of America: ASTM International.

American Society for Testing and Materials. (2018). ASTM-C1611/C1611M-18: Standard Test Method for Slump Flow of Self-Consolidating Concrete. West Conshohocken, PA, United States of America: ASTM International.

Buildings Department; (2006). Available from: http://www.info.gov.hk/bd/english/index.html.

Cachán A., C. (2001). Encuentro Medioambiental Almeriense: En busca de soluciones. (pág. 29). Andalucía.

Criado, M., Fernández-Jiménez, A., \& Palomo, A. (2007). Alkali activation of fly ash: Effect of the $\mathrm{SiO}_{2} / \mathrm{Na}_{2} \mathrm{O}$ ratio Part I: FTIR study. Microporus and Mesoporus Materials, 106.

Cordeiro, G., Toledo-Filho, R., Tavares, L., \& Fairbairn, E. (2009). Ultrafine grinding of sugar cane bagasse ash for application as pozzolanic admixture in concrete. Cement Concrete Research (39), 110115.

Dharanidharan, S., Rajkumar, L., \& Karthick, B. (2015). An experimental study on mechanical properties of concrete by using sugarcane bagasse ash. International Journal of Engineering Sciences \& Research Technology, 401-405.

EFNARC, S. (2002). European Federation of National Associations Representing for Concrete. Especificaciones y directrices para el Hormigón autocompactable - HAC.

Ganesan, K., Rajagopal, K., \& Thangavel, K. (2007). Evaluation of bagasse ash as supplementary cementitious material. Cement and Concrete Composites (29), 515-524. 
Habert G, Arribe D, Dehove T, Espinasse L, Le Roy R. (2012); “Reducing environmental impact by increasing the strength of concrete: quantification of the improvement to concrete bridges". Journal of Cleaner Production. 35: 250-262.

Hill RC, Bowen P. (1997), "Sustainable construction: principles and a framework for attainment". Construction Management Economics. (15): 223-239.

Kovalchuk, G., \& Fernández-Jiménez, A. (2008). Activación alcalina de cenizas volantes. Relación entre el desarrollo mecánico existente y la composición química de la ceniza. Materiales de Construcción, 58.

Martirena, J. F., Middendhort, B., \& Budelmann, H. y. (1997). Estudio de la reacción de hidratación de aglomerantes de cal puzolana fabricados en base a desechos de la industria azucarera. Santa Clara, Cuba: Universidad Central de las Villas-UCLV.

Martirena, J., Middendorf, B., Gehrke, M., \& Budelmann, H. (1998). Use of wastes of the sugar industry as pozzolana in lime-pozzolana binders: study of the reaction. Cement and Concrete Research, 11(28), 1525-1536.

Mehta, P. K. (2000). Avances en la Tecnología del Concreto. (IMCYC, Ed.)

Nasvik, J. (2006). El ABC del concreto autocompactable. (IMCYC, Ed.) Construcción y Tecnología, 50-55.

Neville, A., \& Brooks, J. (1998). Tecnología del concreto. Reimpresión 2010, México: Trillas.

SIAP. (2018). Servicio de Información Agroalimentaria y Pesquera. Recuperado el Enero de 2019, de Servicio de Información Agroalimentaria y Pesquera: http://infosiap.siap.gob.mx:8080/agricola_siap_gobmx/AvanceNacionalSinPrograma.do

Rincón, J., Teixeira, $\quad$ S., $\quad \& \quad$ Romero, M. $\quad$ (2010). http://www.upv.es/contenidos/VALOR10/info/Resumen_005.pdf.

Wadel G. (2014), La sostenibilidad en la construcción industrializada La construcción modular ligera aplicada a la vivienda. Tesis Doctoral. Barcelona (España): Universidad Politécnica de Cataluña.www.tdx.cat/TDX-0122110-180946 (visitada 01/01/14). 